\title{
Conversion of cytochrome $c$ into a peroxidase: Inhibitory mechanisms and implication for neurodegenerative diseases
}

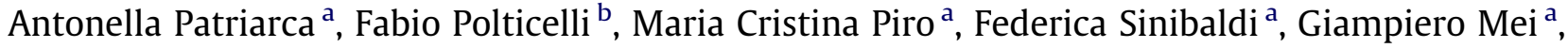 \\ Monica Bari ${ }^{a}$, Roberto Santucci ${ }^{\text {a }}$, Laura Fiorucci ${ }^{\mathrm{a}, *}$ \\ a Department of Experimental Medicine and Biochemical Sciences, University of Rome "TorVergata”, via Montpellier 1, 00133 Rome, Italy \\ ${ }^{\mathrm{b}}$ Department of Biology, University "Roma Tre", Rome, Italy
}

\section{A R T I C L E I N F O}

\section{Article history:}

Received 28 November 2011

and in revised form 6 March 2012

Available online 9 April 2012

\section{Keywords:}

Cytochrome $c$

Peroxidase

ATP

Minocycline

Apoptosis

\begin{abstract}
A B S T R A T
A further function of cytochrome $c$ (cyt $c$ ), beyond respiration, is realized outside mitochondria in the apoptotic program. In the early events of apoptosis, the interaction of cyt $c$ with a mitochondrion-specific phospholipid, cardiolipin (CL), brings about a conformational transition of the protein and acquirement of peroxidase activity. The hallmark of cyt $c$ with peroxidase activity is its partial unfolding accompanied by loosening of the Fe sixth axial bond and an enhanced access of the heme catalytic site to small molecules like $\mathrm{H}_{2} \mathrm{O}_{2}$. To investigate the peroxidase activity of non-native cyt $c$, different forms of the protein were analyzed with the aim to correlate their structural features with the acquired enzymatic activity and apoptogenic properties (wt cyt $c / \mathrm{CL}$ complex and two single cyt $c$ variants, H26Y and Y67H, free and bound to $\mathrm{CL}$ ). The results suggest that cyt $c$ may respond to different environments by changing its fold thus favouring the exertion of different biological functions in different pathophysiological cell conditions. Transitions among different conformations are regulated by endogenous molecules such as ATP and may be affected by synthetic molecules such as minocycline, thus suggesting a mechanism explaining its use as therapeutic agent impacting on disease-associated oxidative and apoptotic mechanisms.
\end{abstract}

(c) 2012 Elsevier Inc. All rights reserved.

\section{Introduction}

Cytochrome $c(\text { cyt } c)^{1}$ acts as an electron transfer protein between cyt $c$ reductase and cyt $c$ oxidase in the mitochondrial respiratory chain. It is a single chain hemoprotein characterized by a relatively high stability due to the covalent attachment of the heme to the polypeptide chain by residues Cys14 and Cys17, while His18 and Met80 act as the Fe ion axial ligands.

A further well-known function of cyt $c$, which is not redox-related, is realized outside mitochondria in the apoptotic program. After its release into the cytosol, cyt $c$ interacts with Apaf-1 protein causing apoptosome formation and subsequent caspase cascade activation. At early stages of apoptosis, a mitochondrion-specific phospholipid, cardiolipin (CL), binds cyt $c$ in-between the outer and the inner mitochondrial membranes. Cyt $c$ bound to CL undergoes a conformational change and acquires peroxidase activity. The catalytic cyt $c$ peroxidizes CL and CL peroxidation products in turn

\footnotetext{
* Corresponding author. Fax: +39 0672596477.

E-mail address: fiorucci@uniroma2.it (L. Fiorucci).

1 Abbreviations used: cyt c, horse heart cytochrome c; CL, cardiolipin; POPC, 1-palmitoy2-oloyl-sn-glycero-3-phosphocholine; Apaf-1, apoptotic protease-activating factor 1; $\mathrm{CD}$, circular dichroism; DPV, differential pulse voltammetry; DTT, 1,4-dithiothreitol; Ac-DEVD-AMC, acetyl-Asp-Glu-Val-Asp-7-amino-4-methylcoumarin.
}

are responsible for membrane permeabilization and cyt $c$ release $[1,2]$.

Thus, the latest function attributed to cyt $c$ has been associated to its ability to be activated and fulfill the task of a peroxidase. The hallmark of cyt $c$ with peroxidase activity is the partial unfolding accompanied by loosening of the Fe sixth axial bond and enhanced access of the heme catalytic site to small molecules like $\mathrm{H}_{2} \mathrm{O}_{2}$.

To investigate the peroxidase activity of cyt $c$ as a function of its conformation, we analyzed different "non native" forms of cyt $c$ with the aim to correlate their structural features with the acquired enzymatic activity. In recent years, several non-native conformers of cyt $c$ were characterized and different biological functions depending on different conformational states of the heme protein were proposed [3]. Whereas native cyt $c$, which is characterized by a compact tertiary structure and six-coordinated state, is a poor peroxidase, CL-bound cyt $c$ shows peroxidase activity and possesses a molten globule-like structure with the Fe-S(Met80) bond disrupted.

In this study, two amino acid residues in cyt $c$ were chosen for mutagenesis in line with the peroxidase mechanism and threedimensional structure of the protein. One was His26, which was replaced by a tyrosine (H26Y) to achieve a protein with a molten globule structure [4]. We hypothesized that the structural 
properties and the partial unfolding of this variant may fulfill the requirements for peroxidase activity. In fact, replacement of histidine with tyrosine frees two loops (20s and 40s loops joined each other by hydrogen bond in the native cyt $c$ ), therefore enhancing protein flexibility and weakening the Fe-S(Met80) bond. The other exchanged aminoacid was Tyr67 that forms a hydrogen bond with the axial ligand Met80 [5] Here it was replaced by a histidine (Y67H), with the aim of introducing a distal histidine in the heme cavity (fundamental element of the peroxidase mechanism). The spectroscopic features of cyt $c / \mathrm{CL}$ complex, H26Y and Y67H mutants (also in the presence of CL) were analyzed and the biological significance of the observed conformational changes was inferred by the relationship with their peroxidase activities and apoptogenic properties. To gain further insights on the capability of ATP to function as allosteric regulator of cyt $c$ conformational transitions [6], its effect on refolding and its modulation of the peroxidase activity of cyt $c$ variants was also investigated.

Moreover, the peroxidase kinetic parameters of the non native conformers here investigated were measured also in the presence of minocycline, an antibiotic endowed with antioxidant properties reported to act as peroxidase inhibitor and neuroprotective agent that delays progression of neuron degeneration [7].

\section{Materials and methods}

Horse heart cytochrome $c$, Peroxidase, Type VI, from Horseradish, Cardiolipin sodium salt $(\mathrm{CL})$, from Bovine Heart, and Minocycline hydrochloride were purchased from Sigma-Aldrich. POPC (1-palmitoyl-2-oleoyl-sn-glycero-3-phosphocholine) was purchased from Avanti Polar Lipids.

\section{Construction of horse cyt c expression system and mutants}

A version of the h-cyt $c$ synthetic gene was designed on the basis of the sequence of a previously reported cyt $c$ synthetic gene [8] and its synthesis accomplished by Primm srl (Milano, Italy). The pBTRI plasmid was converted to the horse cyt $c$ expression plasmid by removing the yeast iso-1-cyt $c$ gene and replacing it with the new synthetic h-cyt $c$ gene. The sequence of the expression construct (pHCyc) was confirmed by DNA sequencing (M-Medical, Milano, Italy). The plasmid pHCyc was then subjected to one round of mutagenesis, which introduced the single H26Y and Y67H substitution into the h-cyt $c$ gene. All mutants and wt h-cyt $c$ expression plasmids were introduced into Escherichia coli JM 109 strain and bacterial expression and purification of the recombinant proteins were conducted as previously described [4]. Recombinant wt h-cyt $c$ was used as control in all the experiments described and reported herein as wt cyt $c$.

\section{Liposome preparation}

Small unilamellar liposomes were prepared by dissolving POPC (1-palmitoy-2-oloyl-sn-glycero-3-phosphocholine) and CL (cardiolipin) (1:1 ratio) in a chloroform/ethanol solution (1:1 vol ratio). The solvents were evaporated under reduced argon atmosphere until a thin film was formed. Complete evaporation was ensured by applying a rotary vacuum pump for at least $2 \mathrm{~h}$. The film was hydrated with a $25 \mathrm{mM}$ Hepes buffer $\mathrm{pH}$ 7.0. After vigorous stirring and 10 freeze and thaw cycles, the liposome suspension was extruded for 31 times thought two stacked membranes with $50 \mathrm{~nm}$ pores.

\section{Circular dichroism spectroscopy}

CD spectra in the far-UV $(210-250 \mathrm{~nm})$ as well in the Soret (400-450 nm) were recorded on a Jasco-710 spectropolarimeter
(Tokyo, Japan) equipped with a PC as data processor and a temperature-controlled holder maintained at $25{ }^{\circ} \mathrm{C}$. Quartz cells with 1and 5-mm light paths were used for measurements in the far-UV and in the Soret region, respectively. The protein samples $(7 \mu \mathrm{M})$ were dissolved in $25 \mathrm{mM}$ Hepes buffer, $\mathrm{pH}$ 7.0.

\section{Absorption spectroscopy}

Absorption measurements were carried out at $25^{\circ} \mathrm{C}$ using a Jasco V-530 spectrophotometer (Tokyo, Japan). Ferric cyt $c$ concentration was determined on the basis of the extinction coefficient $\varepsilon=106 \mathrm{mM}^{-1} \mathrm{~cm}^{-1}$ at $408 \mathrm{~nm}$. To monitor the Fe-S(Met80) bond, optical spectra were recorded in the $650-750 \mathrm{~nm}$ range. Spectra were also recorded in the presence of ATP (concentration range $0-10 \mathrm{mM}$ ) and minocycline (concentration range $0-20 \mu \mathrm{M}$ ).

\section{Tryptophan fluorescence}

The tryptophan fluorescence of H26Y mutant of cyt $c$ in the presence and in the absence of $10 \mu \mathrm{M}$ minocycline was measured at room temperature on a ISS K2 spectrofluorimeter. Samples contained $7 \mu \mathrm{M}$ cyt $c$ in $25 \mathrm{mM}$ Hepes buffer $\mathrm{pH}$ 7.0. Spectra of $7 \mu \mathrm{M}$ wt cyt $c, \mathrm{Y} 67 \mathrm{H}$ mutant and $\mathrm{GdnHCl}$-denatured cyt $c$ were also measured. The excitation wavelength was $293 \mathrm{~nm}$.

\section{Electrochemical measurements}

Voltammetric measurements were carried out at $25^{\circ} \mathrm{C}$ in a glass microcell (sample volume: $1 \mathrm{ml}$ ) equipped with a reference calomel electrode $\left(E=244 \mathrm{mV}\right.$ vs. NHE, at $\left.25^{\circ} \mathrm{C}\right)$, a Pt wire as the counter-electrode. A (2 mm Ø) gold electrode with adsorbed 6mercaptopurine (cyclic voltammetry) was the working electrode used for cyclic voltammetry measurements; a ( $3 \mathrm{~mm} \mathrm{Ø)} \mathrm{pyrolytic}$ graphite electrode was instead employed for differential pulse voltammetry measurements. All the electrodes used were purchased from Amel (Milan, Italy). An Amel 433/W multipolarograph, interfaced with a PC, was employed for electrochemical measurements. Before measurements, the solution was deaerated for $30 \mathrm{~min}$ by a gentle flow of pure nitrogen maintained just above the solution surface. Further measurements were performed using a procedure for the chemical modification of the gold electrode as previously described [9]. Briefly, the electrode was dipped in a saturated aqueous (6-mercaptopurine) solution for $15 \mathrm{~min}$. The electrode was then rinsed with distilled water and dipped in the electrochemical cell containing the protein $\mu \mathrm{M}$ ) solubilized in $20 \mathrm{mM}$ Hepes, $\mathrm{pH}$ 7.0, $+\mathrm{NaClO}_{4}$

\section{Steady-state kinetic studies}

Peroxidase activity of free wt cyt $c$, cyt $c / \mathrm{Cl}$ complex, H26Y, $\mathrm{Y} 67 \mathrm{H}$ and $\mathrm{Y} 67 \mathrm{H} / \mathrm{CL}$ complex was determined by measuring the $\mathrm{H}_{2} \mathrm{O}_{2}$-dependent oxidation of guaiacol using guaiacol at different concentrations. Peroxidase activity was estimated $2 \mathrm{~min}$ after $\mathrm{H}_{2} \mathrm{O}_{2}$ treatment. The steady-state kinetics of oxidation of guaiacol to give its tetramer and the inhibition effect of minocycline on peroxidase activity was measured spectrophotometrically at $470 \mathrm{~nm}$ at $25{ }^{\circ} \mathrm{C}\left(\varepsilon_{470}=26.6 \mathrm{mM}^{-1} \mathrm{~cm}^{-1}\right)$. The data were fitted using non linear regression analysis according to the Michaelis-Menten equation and by using the Lineweaver-Burk equation. For the determination of inhibition constants $\left(K_{i}\right)$, the kinetic results were plotted according to the method of Cornish-Bowden [10]. To further characterize the type of inhibition, secondary plots were used [11]. The effect of ATP on the peroxidase activity of cyt $c / C L$ complex and H26Y mutant was also measured in the presence of increasing concentration of ATP (concentration range $0-10 \mathrm{mM}$ ). 

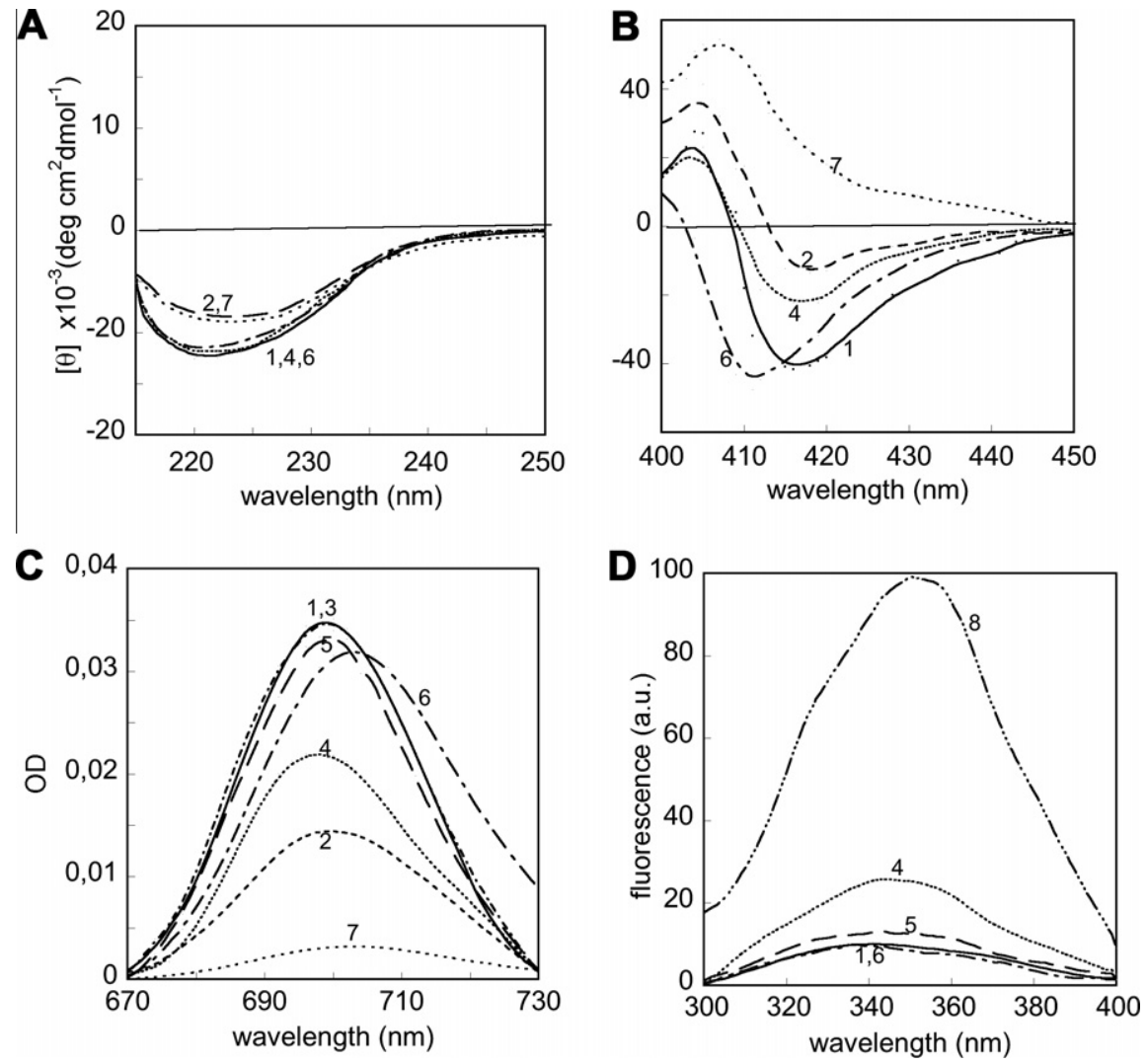

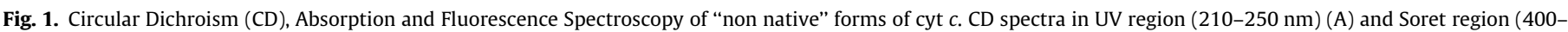

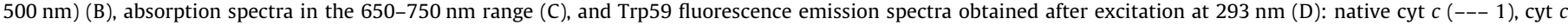

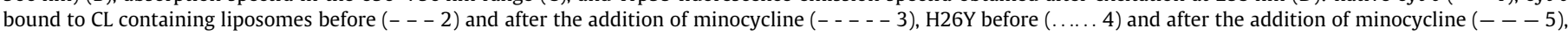

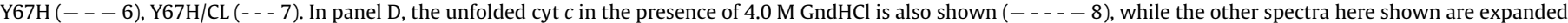
2-fold. Protein samples $(7 \mu \mathrm{M})$ were dissolved in $25 \mathrm{mM}$ Hepes $(\mathrm{pH} 7.0)$ at $25^{\circ} \mathrm{C}$.

\section{Docking simulations of minocycline onto cyt c}

Docking simulations of minocycline onto the oxidized horse heart cyt $c$ three-dimensional structure (PDB code 1AKK; [12] were performed using Autodock Vina [13], a new version of the docking software Autodock which improves the speed and accuracy of docking with a new scoring function, efficient optimization and multithreading. The exhaustiveness of the docking search was progressively increased in successive runs from a value of 8 (default value) to a value of 30 , until the same lowest energy complex, represented in Fig. 3, was found in two subsequent docking runs.

\section{Cell-free assays for caspase activity}

The pro-apoptotic activity of wt cyt $c$ and variants was assessed by their ability to activate caspases present in cytosolic extracts (S100). The S-100 extract prepared from U937 cells (100 $\mu \mathrm{g}$ of proteins) was incubated for $1 \mathrm{~h}$ with $5 \mu \mathrm{M}$ cyt $c$ (wild type, H26Y, $\mathrm{Y} 67 \mathrm{H}$ ), in lysis buffer and in the presence of $1 \mathrm{mM}$ ATP and minocycline at various concentrations as indicated) at $25^{\circ} \mathrm{C}$. After incubation, $1 \mathrm{mM}$ DTT and $25 \mu \mathrm{M} \mathrm{N}$-acetyl-DEVD-AMC was added to measure the activity of caspase-3. Hydrolysis of the substrate was followed fluorometrically at $380 \mathrm{~nm}$ (excitation) and $460 \mathrm{~nm}$ (emission) for the caspase-3 substrate AC-DEVD-AMC.

\section{Immunoblotting}

Ten microliter of extracts (about $10 \mu \mathrm{g}$ total proteins) were incubated for $1 \mathrm{~h}$ with $5 \mu \mathrm{M}$ cyt $c$ (wt, H26Y, Y67H and cyt $c / \mathrm{CL}$ complex) in lysis buffer and in the presence of $1 \mathrm{mM}$ ATP and
$20 \mu \mathrm{M}$ minocycline (as indicated) at $25^{\circ} \mathrm{C}$. After incubation, the samples were subjected to a $10.5 \%$ SDS-PAGE and transferred to a nitrocellulose filter. Membrane was blocked overnight in $50 \mathrm{mM}$ Tris ( $\mathrm{pH} 8.0$ ) containing $500 \mathrm{mM} \mathrm{NaCl}$ and 3\% dried milk (blocking solution, BS); then it was probed $3 \mathrm{~h}$ with anti-caspase 3 (rabbit polyclonal antibodies) in BS, followed by $1 \mathrm{~h}$ with the secondary IgG. The blots were stained by the "Kit chemiluminescent peroxidase substrate" (Sigma), according to the manufacturer's instructions.

\section{Results and discussion}

Structural and electrochemical characterization of "non native" conformations of cyt $c$

The structural characterization of wt cyt $c$, cyt $c / C L$ complex $\mathrm{H} 26 \mathrm{Y}$, and $\mathrm{Y} 67 \mathrm{H}$ mutants (also complexed with $\mathrm{CL}$ ) was performed by CD, Absorption and Fluorescence Spectroscopy. In this work, we used recombinant horse cyt $c$ as control (wt cyt $c$ ). Stability, spectroscopic and functional features of recombinant horse cyt $c$ and horse cyt $c$ from heart are nearly identical (data not shown). Furthermore, Fe of heme in all cyt $c$ forms is always in the oxidized state $(\mathrm{Fe}+3)$. CD spectra in the far-UV (210$250 \mathrm{~nm}$ ) of wt cyt $c$, wt cyt $c$ bound to liposomes, and cyt $c$ mutants (H26Y, Y67H) (Fig. 1A), show the typical features of $\alpha$-helical protein structure. A dramatic loss of $\alpha$-helical structure content was observed in H26Y/CL structure (data not shown), suggesting a strong unfolding effect of CL on the H26Y mutant characterized in itself by a "non native" tertiary structure. Spectra of wt cyt $c$ 
and $\mathrm{Y} 67 \mathrm{H}$ bound to $\mathrm{CL}$-containing liposomes show a reduction of the CD signal at $222 \mathrm{~nm}$ suggesting a slight decrease of the $\alpha$-helical content. The possibility that such decrease would correspond to an increase of $\beta$-sheet content, as discussed by Spiro and coworkers [14], cannot be ruled out. Wt cyt $c$ CD spectrum in the Soret region (400-500 $\mathrm{nm}$ ) shows a major positive and negative Cotton effects at $404 \mathrm{~nm}$ and $416 \mathrm{~nm}$, respectively. The intensity of the $416 \mathrm{~nm}$ band depends on the heme-vicinal residues interaction and decreases when the distance and orientation of the Phe 82 side chain, positioned on the Met80 side of the plane, are perturbed [15] It is considered diagnostic for the integrity of the Met80-Fe(III) axial bond [16]. Thus, reduction of the CD signal observed for cyt $c$ bound to liposomes and for H26Y mutant (Fig. 1B) suggests the displacement of Met80 from the axial coordination of the heme-iron. The replacement of Tyr67 with histidine in the distal pocket of heme cavity, results in a CD with decreased positive Cotton effect and negative Cotton effect with similar amplitude and blue shifted at $414 \mathrm{~nm}$ with respect to native cyt $c$, still suggesting the stability of the coordination of the Met80 to the iron of the heme (Fig. 1B). After binding to CL, the Soret $\mathrm{CD}$ Y67H spectrum is converted to a single positive band with a maximum near $408 \mathrm{~nm}$. Such spectral changes indicate a strong conformational change in the heme pocket.

According to these findings, the absorption spectra in the 650$750 \mathrm{~nm}$ range show a decrease of the $695 \mathrm{~nm}$ band amplitude for the cyt $c / C L$ complex and H26Y mutant and not for Y67H mutant. For the last mutant, a complete loss of the $695 \mathrm{~nm}$ band is observed when it binds CL. In the current study, we have used the analysis of the absorption band at $695 \mathrm{~nm}$ to assess the conformational transitions in cyt $c$ associated to a partial unfolding evidenced as decrease or loss of the $\mathrm{Fe}-\mathrm{S}$ (Met80) bond as previously suggested $[17,18]$. Interestingly, the reduction of the absorbance at $695 \mathrm{~nm}$ shown in the electronic spectra of H26Y mutant and cyt $c$ bound to $\mathrm{CL}$ liposomes is no longer detectable in the presence of minocycline (Fig. 1C). Analysis of the electronic spectrum of Y67H mutant shows a shift by $2 \mathrm{~nm}$ of the absorption band at $695 \mathrm{~nm}$ that may be indicative of a slightly modified heme electronic structure, as already described for the mutation Y67F on rat cyt $c$ [19]. As indicated by the amplitude of the $695 \mathrm{~nm}$ band, only a small effect, if any, is induced by mutation of Tyr67 on the Fe-S(Met80) bond integrity (Fig. 1C), in agreement with previous results [20]. Tyr67 forms a hydrogen bond network with the axial ligand Met80, Asn52, and Thr78 [5] and our results suggest that mutation of this residue brings about a rearrangement of hydrogen bonds that does not affect the heme-Met80 ligation. Nevertheless, Fe-S(Met80) bond seems to be absent in $\mathrm{Y} 67 \mathrm{H} / \mathrm{CL}$ complex. Wt cyt $c$ is characterized by efficient quenching of Trp59 fluorescence due to the closely located heme group. The fluorescence spectra of Y67H mutant is similar to wt cyt $c$ while the fully unfolded cyt $c$ obtained upon addition of $4.0 \mathrm{M} \mathrm{GdnHCl}$ shows a strong Trp59 fluorescence with a longer wavelength maximum consistent with a more polar tryptophan environment (Fig. 1D). The H26Y mutant shows a larger fluorescence quantum yield with respect to the wild type, consistent with a decreased tertiary packing of tryptophan related to the increased structure flexibility in the heme pocket region discussed above. Such structure flexibility seems to be regulated by interaction of the H26Y mutant with minocycline (Fig. 1D). Upon binding of minocycline, heme quenches the Trp59 fluorescence again suggesting a contribution of minocycline to the rigidity of $\mathrm{H} 26 \mathrm{Y}$ structure.

A previous investigation on the H26Y mutant has suggested that His26 plays a critical role for protein stabilization [4]. The hydrogen bond between His26 and the backbone carbonyl of Pro44 keeps the 20 s and the 40 s $\Omega$-loops joined together and sterically close. The flexibility of the H26Y variant may reduce the native $\mathrm{Fe}-\mathrm{S}$ (Met80) axial bond strength. The mutation-induced conformational changes (of tertiary nature) occurring in the pro- tein, which involve the heme pocket region, may provoke a higher exposure of the heme group to the solvent. To support the hypothesis, we investigated the redox property of the mutant. Differential pulse voltammetry (DPV) applied to the H26Y mutant (no signal was in fact obtained using the more rapid technique, cyclic voltammetry) identifies a well defined wave centered at -150 $( \pm 5) \mathrm{mV}$ vs SCE. The calculated redox potential, $E_{1 / 2}=54( \pm 8) \mathrm{mV}$ vs NHE, is significantly lower than that typical of the native protein $\left(E_{1 / 2}=250 \mathrm{mV}\right.$ vs NHE), confirming the less-solvent shielding of the heme due to the increased polypeptide flexibility and suggesting a stabilization of the ferric heme of the variant. Cyclic voltammetry applied to the $\mathrm{Y} 67 \mathrm{H}$ mutant shows voltammograms characterized by cathodic and anodic peaks similar in shape and magnitude (not shown). The calculated redox potential is higher than that of the native protein $\left(E_{1 / 2} \cong 410 \mathrm{mV}\right.$ vs. NHE). Since the spectroscopic properties of the $\mathrm{Y} 67 \mathrm{H}$ mutant are not so different from the native protein, the high value of redox potential detected may result from a stabilization of the reduced form with respect to the oxidized one owing to the introduction of a positive charge next to heme iron.

\section{Induction and inhibition of peroxidase activity}

Assessment of peroxidase activity was performed using $1 \mathrm{mM}$ $\mathrm{H}_{2} \mathrm{O}_{2}$. This, $\mathrm{H}_{2} \mathrm{O}_{2}$ concentration was required to optimize product formation and it does not affect protein and heme integrity as checked by the absorbance profile in the Soret region of wild type and modified cytochromes $c$ here analyzed (data not shown). In cyt $c$, the heme iron forms two axial bonds: one with His18 on the proximal side of heme and one with Met80 on the distal side. The bond with Met80 hinders the iron interactions with $\mathrm{H}_{2} \mathrm{O}_{2}$. However, under conditions inducing dissociation of FeS(Met80), the properties of cyt $c$ result significantly changed. Partial unfolding of the protein causes an enhancement of the peroxidase activity of cyt $c$. Steady-state rates at various concentrations of guaiacol were analyzed by fitting to the Michaelis-Menten equation by using non linear regression analysis and the Lineweaver-Burk equation. Data obtained from both models were in good agreement. The kinetic parameters of guaiacol oxidation by cyt $c / C L$ complex, H26Y, Y67H, and Y67H/CL mutants were measured also in the presence of minocycline. ForY67H mutant, the peroxidase activity is only slightly increased with respect to the wild type, in agreement with spectroscopic data that showing no remarkable perturbation of the heme-Met80 bond (see Table 1). Also in the Y67H/CL complex, no enhancement of peroxidase activity was observed, probably due to a protein conformational change in the heme pocket yielding a long distance between the heme iron and histidine and a poor "peroxidase active site". This situation has also been observed in the peroxidase activities of myoglobin mutants [21]. On the other hand, cyt c/CL complex and H26Y mutant both show a remarkable enhancement of peroxidase activity with respect to wt cyt $c$. The major contribution to the increase of $k_{\text {cat }} /$ $K_{m}$ ratio is due to lower $K_{m}$ values, i.e. to the higher affinity of guaiacol towards both cyt $c$ variants or alternatively towards compound I that forms in the peroxidase catalytic cycle when heme loses the sixth axial ligand. The inverse plots of the steady-state rates of oxidized guaiacol formation for the cyt $c / C L$ complex and H26Y mutant (Fig. 2A and C) in the absence and in the presence of increasing minocycline concentrations were linear and parallel, suggesting that minocycline is an uncompetitive inhibitor of guaiacol oxidation. $K_{i}$ values were calculated to be $1.0 \pm 0.1 \mu \mathrm{M}$ and $10.1 \pm 1.1 \mu \mathrm{M}$ for the H26Y mutant and cyt $c / \mathrm{CL}$ complex, respectively (Fig. $2 \mathrm{~B}$ and D). By replotting $1 /$ i0.5 values (concentration of inhibitor that produces $50 \%$ inhibition) against v/V (the uninhibited rate divided by the limiting rate), lines passing through origin were obtained, thus confirming the strictly uncompetitive 
Table 1

Kinetics parameters of peroxidase activity of wt cyt $c$ and "non native" forms. The standard deviations are given.

\begin{tabular}{|c|c|c|c|c|c|}
\hline Protein & $K_{m}(\mu \mathrm{M})$ & $k_{\text {cat }}\left(\mathrm{s}^{-1}\right)$ & $k_{\text {cat }} / K_{m}\left(\mathrm{M}^{-1} \mathrm{~s}^{-1}\right)$ & $K_{i}(\mu \mathrm{M})$ & Type of inhibition \\
\hline Wt cyt $c$ & $23 \pm 1.4$ & $0.014 \pm 0.001$ & $609 \pm 15$ & No inhibition & - \\
\hline $\mathrm{Y} 67 \mathrm{H}^{[\mathrm{a}]}$ & $24 \pm 2.8$ & $0.020 \pm 0.002$ & $833 \pm 23$ & No inhibition & - \\
\hline $\mathrm{H} 26 \mathrm{Y}^{[\mathrm{a}]}$ & $11 \pm 0.8$ & $0.034 \pm 0.001$ & $2700 \pm 144$ & $1.0 \pm 0.1$ & Uncompetitive \\
\hline cyt $c / \mathrm{CL}^{[\mathrm{a}]}$ & $6.2 \pm 2.0$ & $0.020 \pm 0.002$ & $3225 \pm 216$ & $10 \pm 1.1$ & Uncompetitive \\
\hline $\mathrm{Y} 67 \mathrm{H} / \mathrm{CL}^{[\mathrm{a}]}$ & $26 \pm 2.9$ & $0.021 \pm 0.002$ & $808 \pm 24$ & No inhibition & \\
\hline $\mathrm{HPR}^{[\mathrm{b}]}$ & $3300 \pm 500$ & $562 \pm 43$ & $170,000 \pm 3500$ & No inhibition & - \\
\hline
\end{tabular}

Reaction conditions: [a] $1 \mu \mathrm{M}$ protein, $1 \mathrm{mM} \mathrm{H}_{2} \mathrm{O}_{2}$, and $25 \mathrm{mM}$ Hepes $\mathrm{pH} 7,0$ at $25^{\circ} \mathrm{C}$ [b] as above but 2 nM horse radish peroxidase (HRP) as enzyme.

cyt c / CL complex
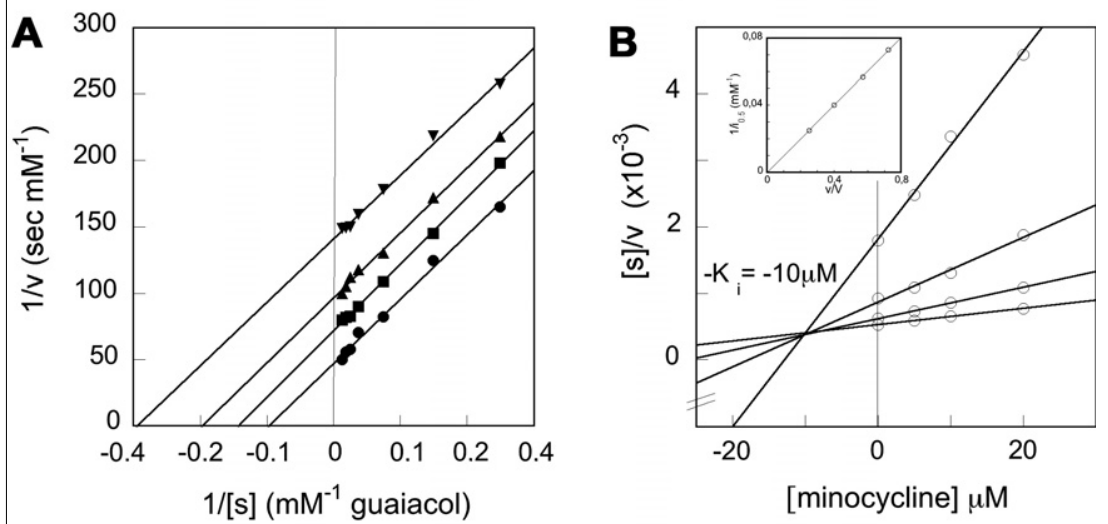

$\mathrm{H} 26 \mathrm{Y}$
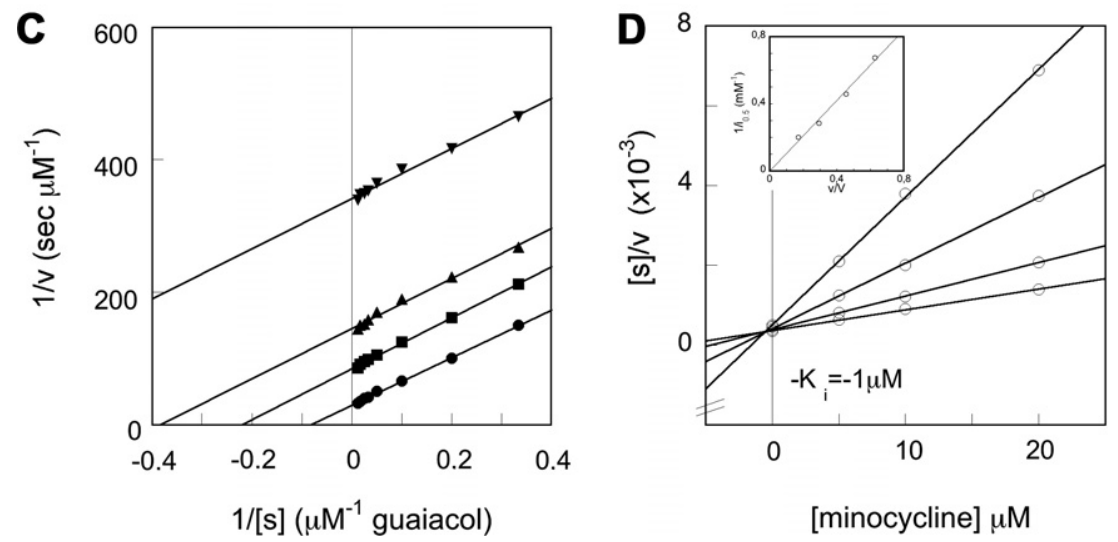

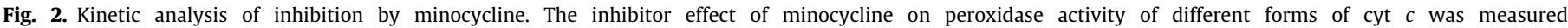

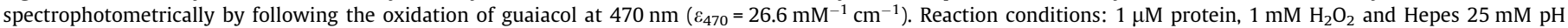

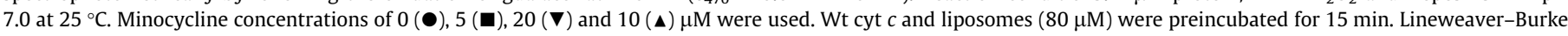

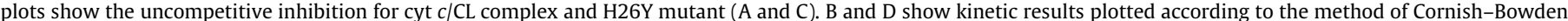

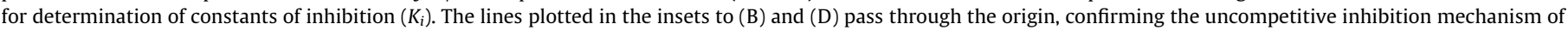
minocycline.

behaviour. The finding that minocycline inhibits guaiacol oxidation with an uncompetitive mechanism suggests the presence of specific sites on cyt $c$ for minocycline interaction that might directly modulate the accessibility of $\mathrm{H}_{2} \mathrm{O}_{2}$ and/or guaiacol to the heme site. Indeed, such interaction seems to induce conformational changes affecting the $\mathrm{Fe}-\mathrm{S}$ (Met80) bond integrity as supported by the results of the electronic absorption spectra described above.

It is worthy to note that, during apoptosis, CL migrates from the inner leaflet to the outer leaflet of the inner mitochondrial membrane, making easier the interaction with cyt $c$ and the conversion of cyt $c$ into a peroxidase $[2,18,22]$. Gaining of enzymatic activity at this stage could be responsible for the oxidation of vulnerable cytosolic proteins such as $\alpha$-synuclein that associates in large aggregates leading to the formation of Lewy bodies that were found in the degenerating neurons from subjects with Parkinson's disease [23]. Of particular interest is the presence of a considerable amount of immunoreactive cyt $c$ in Lewy bodies [7]. Taken as a whole, the evidence leads us to propose that the cyt c's conversion into a peroxidase in the early events of apoptosis may play a significant role in the complex neurodegeneration scenario. as it was hypothesized for a few heme-peroxidases [7] Thus it would be of relevance, relying on structural studies, to identify molecules able to regulate the peroxidase function of cyt $c / C L$ complex and thus functioning as potential inhibitors of CL oxidation with anti-apoptotic properties. To this aim, Kagan et al. recently described new approaches to mitochondrial targeting of small biologically active 


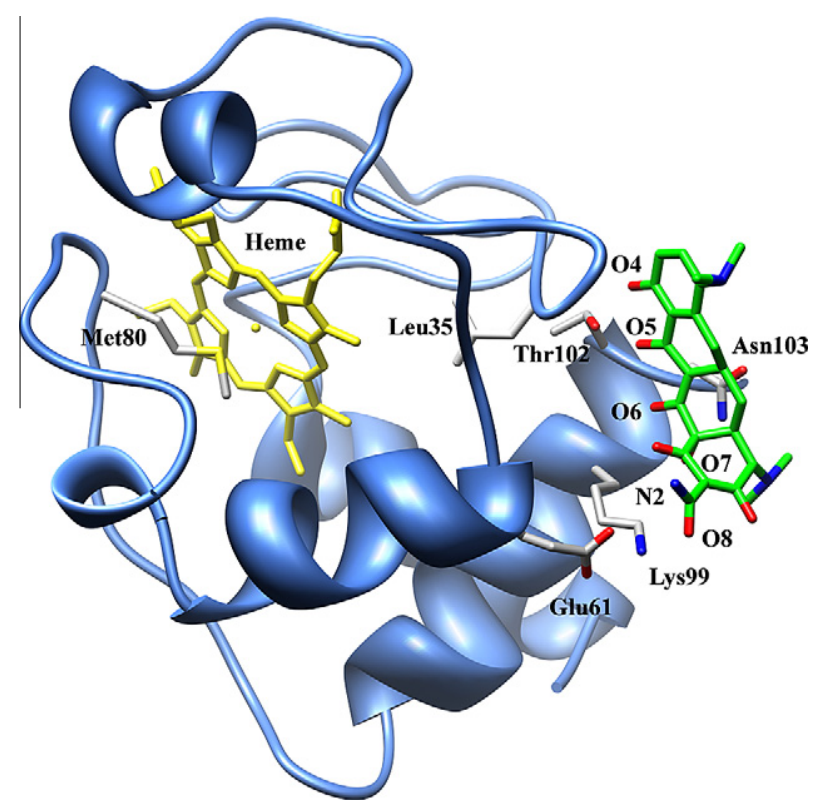

Fig. 3. Docking simulations of minocycline onto cyt $c$ Schematic representation of the cyt $c$-minocycline putative complex has been obtained by docking simulations using Autodock Vina (16). For clarity, only the heme moiety, Met80, minocycline and the residues interacting with it (Leu35, Glu61, Lys99, Thr102 and Asn103) are shown. For clarity of representation minocycline carbon atoms are shown in green. (For interpretation of the references to colour in this figure legend, the reader is referred to the web version of this article.)

molecules [24]. Our findings show that minocycline binds cyt $c$ and regulates its peroxidase activity. Minocycline has long been proved to be absorbed well into CNS and it is used in clinical trials in several neurodegenerative diseases. Thus, the capability of minocycline to affect the peroxidase activity of cyt $c$ may be proposed as a biochemical mechanism involved along with other factors and in combination with other mechanisms, in its neuroprotective effect. [25-27].

\section{Docking simulations of minocycline onto cyt c}

Fig. 3 shows the putative cyt $c$-minocycline complex obtained by flexible docking simulations using Autodock Vina [13]. In this complex, minocycline binds in a depression of the cyt $c$ surface adjacent to the loop encompassing residues 30-46 which encloses and shields from solvent the heme group. In this complex minocycline polar groups establish several hydrogen bonds/electrostatic interactions with sidechains and backbone groups of cyt $c$, detailed in Table 2. The binding energy calculated by the Autodock Vina scoring function is $-6.6 \mathrm{kcal} / \mathrm{mol}$, corresponding to a $K_{d}$ of $15.5 \mu \mathrm{M}$, a value in good agreement with the experimentally determined $K_{i}$ value $(10 \mu \mathrm{M})$. As can be seen from Table 2 and Fig. 3, these interactions link different regions of the cyt $c$ structure and thus are expected to stabilize the whole structure of cyt $c$ in the native conformation and to prevent binding of lipids in the vicinity of heme. This hypothesis is in line with the observation of an uncompetitive inhibition of the peroxidase activity of the cyt $c / C L$ complex. In fact minocycline, similarly to ATP, would revert the non native conformation of the protein to the native (lipid-free) one, thus lowering heme accessibility to $\mathrm{H}_{2} \mathrm{O}_{2}$.

The effect of minocycline on the structural changes caused by CL binding to cyt $c$ is in agreement with the observation that minocycline can revert also the effects caused by H26Y mutation. The interaction of minocycline in the cyt $c$ region adjacent to the $40 \mathrm{~s}$ omega-loop may enhance the overall rigidity and stability of the
Table 2

Hydrogen bonds/electrostatic interactions observed in the putative mynocycline-cyt $c$ complex obtained by docking simulations.

\begin{tabular}{lll}
\hline Minocycline & cyt $c$ & Distance $(\AA)$ \\
\hline $\mathrm{N} 2$ & Glu61:Oع2 & 3.5 \\
O8 & Lys99: $\mathrm{N} \varepsilon$ & 3.2 \\
O6 & Asn103:N $\delta 2$ & 2.7 \\
O7 & Asn103:N $\delta 2$ & 2.9 \\
O5 & Thr102:O $\gamma 1$ & 2.8 \\
O4 & Leu35:O & 3.4 \\
O4 & Leu35:N & 3.2 \\
\hline
\end{tabular}

protein, in spite of the absence of the hydrogen bond that keeps the $20 \mathrm{~s}$ and the $40 \mathrm{~s} \Omega$-loops joined together. Again, this hypothesis is confirmed by the uncompetitive nature of minocycline inhibition of $\mathrm{H} 26 \mathrm{Y}$ cyt $c$ peroxidase activity.

The relevant conformational changes and the partial unfolding occurring in cyt $c$ when the protein binds to CL $[18,28]$ may be reverted. We previously reported that ATP disrupts the oleic acid-cyt $c$ interaction by binding to a specific site of the protein; thus the nucleotide regulates the cyt $c$ conformational transitions [6,17]. Here we show that ATP acts also on the cyt $c / C L$ complex by inducing complex dissociation. In parallel, ATP abolishes the peroxidase activity of the cyt $c / C L$ complex (Fig. 4A). It is likely that ATP competes with the CL head groups for a binding site on cyt $c$ that comprises basic residues, such as Lys88 and Arg91 previously proposed to form the ATP binding site [6,28]. Although ATP seems to behave as a regulator of the conformational transitions and of the peroxidase activity also in the case of H26Y mutant, in this case its effect results lower since the complete recovery of the properties typical
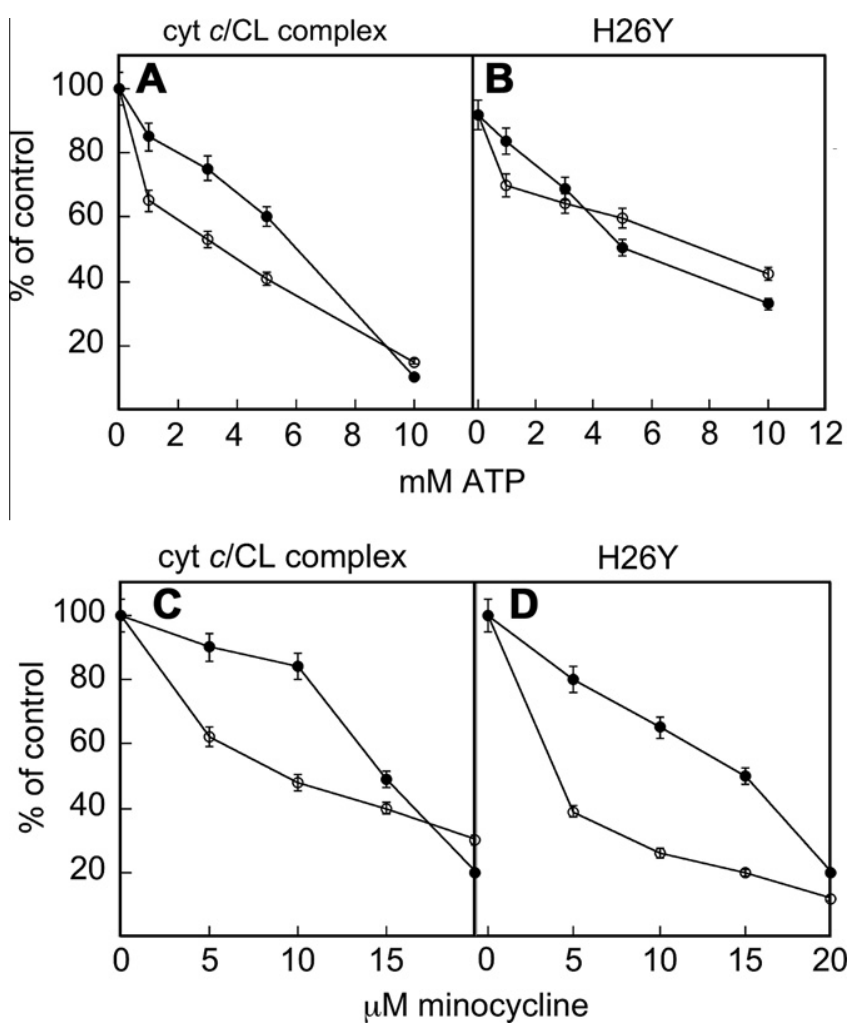

Fig. 4. Effect of ATP and minocycline. Effect of ATP (A and B) and minocycline (C and $\mathrm{D}$ ) on peroxidase activity (empty circles) and on the absorption band loss at $695 \mathrm{~nm}$ (full circles) of cyt c/CL complex (A and C) and H26Y (B and D). Peroxidase activity was measured with wt cyt $c(1 \mu \mathrm{M})$, liposomes $(80 \mu \mathrm{M})$, guaiacol $(40 \mu \mathrm{M})$, $\mathrm{H}_{2} \mathrm{O}_{2}(1 \mathrm{mM})$ in the presence of increasing concentrations of ATP and minocycline, in Hepes $25 \mathrm{mM}$ pH 7.0 at $25^{\circ} \mathrm{C}$. 


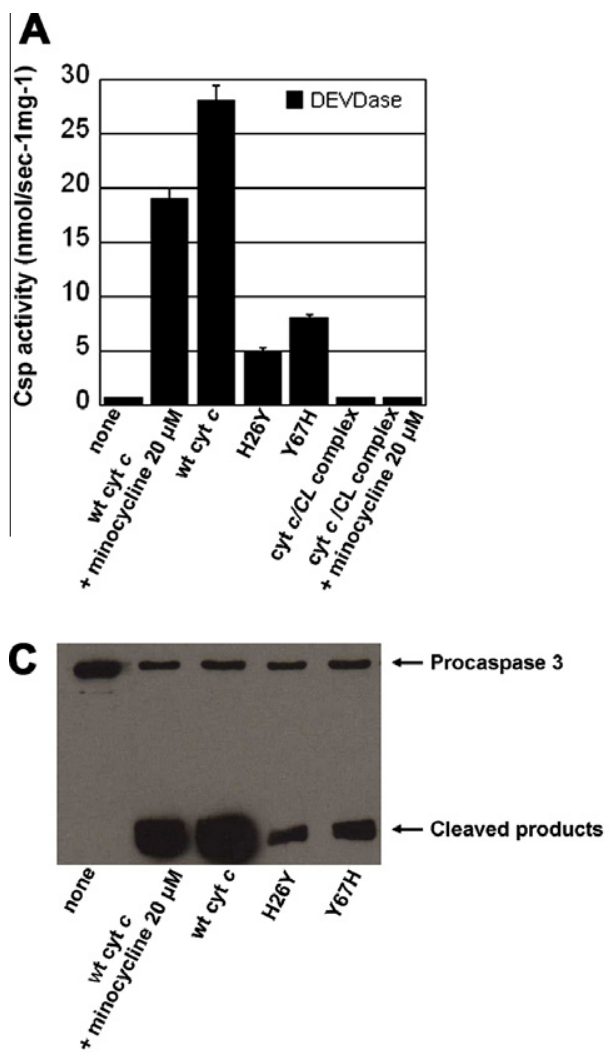

B

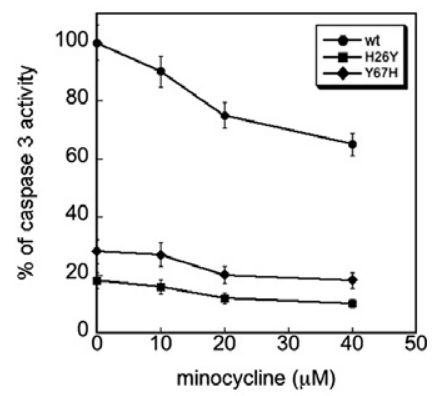

D

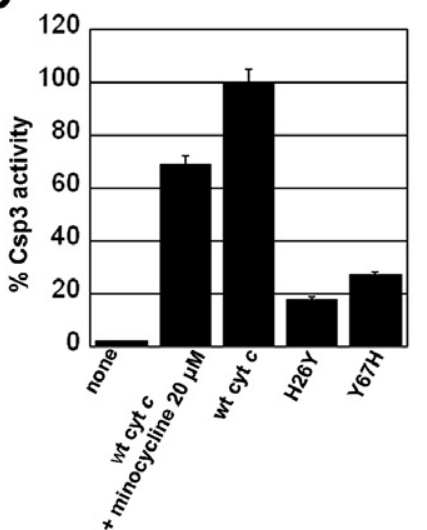

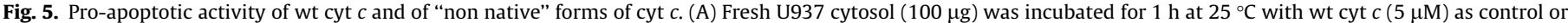

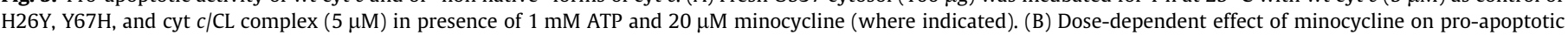

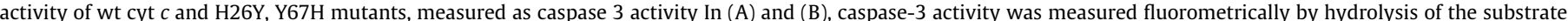

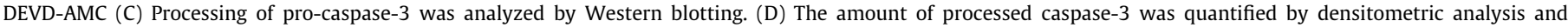
expressed as a percentage of control to which a value of 100 has been given.

of the unbound protein (as the integrity of Fe-S(Met80) bond and poor peroxidase activity) were not observed at the physiological cellular ATP concentration values tested here (1-10 mM) (Fig. 4B). Of note, minocycline in the concentration range 1$20 \mu \mathrm{M}$ is effective in reducing the loss of $695 \mathrm{~nm}$ signal and peroxidase activity of the H26Y mutant (Fig. 4D). Comparison of the inhibitory profile of minocycline and ATP at different concentrations on conformational change and peroxidase activity of cyt $c$ induced by binding to CL and on the partially "unfolded" $\mathrm{H} 26 \mathrm{Y}$ mutant (Fig. 4C and D), makes clear that minocycline distinguishes at a larger extent with respect to ATP the recovery of the Fe$\mathrm{S}$ (Met80) integrity from the peroxidase activity. It may be argued that minocycline binding to cyt $c$ is able to stabilize unfolding intermediates invisible in optical measurements but endowed with peroxidase activity.

\section{Caspase activation in cell-free system}

The apoptotic activity of cyt $c$ variants has been compared with that of wt cyt $c$ by using a cell-free activation assay. As shown in Fig. 5A, addition of exogenous $5 \mu \mathrm{M}$ wt cyt $c$ and $1 \mathrm{mM}$ ATP to an in vitro system of cytosolic fractions from U937 cells, induces caspase activation, as measured by using the caspase-3-specific AcDEVD-AMC fluorogenic substrate. Addition of minocycline brings about a moderate but significant concentration-dependent caspase activity decrease (Fig. 5B). Thus, the neuroprotective effect of minocycline shown in pre-clinical studies [27] may be related to the downregulation of neuronal death that represents a hallmark of many neurodegenerative diseases.

Significantly, the substitution of histidine with tyrosine residue at position 26 (in the $\mathrm{H} 26 \mathrm{Y}$ mutant) brings about a reduction of the protein proapoptotic capability measured as caspase 3 activity (Fig. 5A). As said, the H26Y mutation induces the rupture of the hydrogen bond between the 20 s and 40 s $\Omega$-loops; the major flexibility of the two loops affects the heme pocket structure, weakening the native $\mathrm{Fe}-\mathrm{S}$ (Met80) bond [29]. Such conformational change is sufficient to hamper the electrostatic interaction of cyt $c$ with Apaf-1, which is mediated by the binding of positively charged lysine residues lying on cyt $c$ loops to negatively charged aspartate residues on Apaf-1 [30]. On the other hand, the high redox potential of the $\mathrm{Y} 67 \mathrm{H}$ mutant, interpreted as a mutation-induced stabilization of the reduced state of the protein, provides a rationale for the low proapoptotic action shown by the Y67H mutant (Fig 5A). It is well known that cyt $c$ carries out proapoptotic activity in the oxidized state and that changes in conformation which are a result of its redox state might switch on/off the apoptosome formation [6, 31]. While, cyt $c$ is released from mitochondria almost certainly in the oxidized state, it will be reduced by cytoplasmic GSH. No caspase 3 activity was observed for the cyt $c / C L$ complex suggesting the inability of cyt $c$ bound to CL to form the apoptosome, also in the presence of minocycline, whose binding to cyt $c$ probably interferes with cyt c-Apaf-1 interaction. Accordingly, a small dose-dependent effect of minocycline on caspase activity of H26Y and $\mathrm{Y} 67 \mathrm{H}$ mutants is also detectable (Fig. 5B). The caspase-3 activation data, as indicated by DEVDase activity measurement, were confirmed by Western blot detection of procaspase- 3 cleavage and by densitometric analysis (Fig. 5C and D).

\section{Conclusions}

Taken together, these observations suggest that cyt $c$ may respond to different environments by changing its fold and that this 
process allows it to exert different biological functions in different cell physiological and pathophysiological conditions. Transitions among different conformations are regulated by endogenous molecules such as ATP and may be affected by synthetic molecules such as minocycline. Minocycline has been shown to have neuroprotective effects in animal models of neurodegenerative diseases $[7,25,26]$. Our findings suggest as minocycline may have a twofold effect on cyt $c$ apoptotic role: a former effect due to its potency in avoiding or decreasing the peroxidase activity of cyt $c$ in the early stages of apoptosis and a later effect related to its competition in cyt $c$-Apaf- 1 binding interaction in the cytosol. Such evidence may give a contribution to the research on minocycline that is used as a therapeutic agent impacting on disease-associated oxidative and apoptotic mechanisms such as neurodegenerative diseases. The multi-faceted effects exerted by minocycline need further studies to unravel the molecular mechanisms (probably disparate) involved in its action.

\section{Acknowledgments}

We gratefully acknowledge the financial support of the Italian Ministry of University and Research (MIUR) through PRIN 2007KAWXCL. We thank Dr. L. Stella for helpful advice for liposome preparations.

\section{References}

[1] S. Orrenius, V. Gogvadze, B. Zhivotovsky, Annu. Rev. Pharmacol. Toxicol. 47 (2007) 143-183.

[2] V.E. Kagan, V.A. Tyurin, J. Jiang, Y.Y. Tyurina, V.B. Ritov, A.A. Amoscato, A.N Osipov, N.A. Belikova, A.A. Kapralov, V. Kini, I.I. Vlasova, O. Zhao, M. Zou, P. Di, D.A. Svistunenko, I.V. Kurnikov, G.G. Borisenko, Nat. Chem. Biol. 4 (2005) 223 232.

[3] R. Santucci, F. Sinibaldi, L. Fiorucci, Mini Rev. Med. Chem. 8 (2008) 57-62.

[4] F. Sinibaldi, M.C. Piro, B.D. Howes, G. Smulevich, F. Ascoli, R. Santucci, Biochemistry 42 (2003) 7604-7610.

[5] A.M. Berghuis, J.G. Guillemette, M. Smith, G.D. Brayer, J. Mol. Biol. 235 (1994) 1326-1341.

[6] A. Patriarca, T. Eliseo, F. Sinibaldi, M.C. Piro, R. Melis, M. Paci, D.O. Cicero, F. Polticelli, R. Santucci, L. Fiorucci, Biochemistry 48 (2009) 3279-3287.
[7] J. Everse, P.W. Coates, Neurobiol. Aging 30 (2009) 1011-1025

[8] N.C. Patel, M.C. Lind, G.J. Pielak, Protein Expr. Purif. 22 (2001) 220-224.

[9] R. Santucci, C. Bongiovanni, G. Mei, T. Ferri, F. Polizio, A. Desideri, Biochemistry 39 (2000) 12632-12638.

[10] A. Cornish-Bowden, Biochem. J. 137 (1974) 143-144.

[11] A. Cortes, Biochem. J. 357 (2001) 263-268.

[12] L. Banci, I. Bertini, H.B. Gray, C. Luchinat, T. Reddig, A. Rosato, P. Turano, Biochemistry 36 (1997) 9867-9877.

[13] O. Trott, A.J. Olson, J. Comp. Chem. 31 (2010) 455-461.

[14] G. Balakrishnan, Y. Hu, O.F. Oyerinde, J. Su, J.T. Groves, T.G. Spiro, J. Am. Chem. Soc. 129 (2007) 504-505.

[15] G.J. Pielak, K. Oikawa, A.G. Mauk, M. Smith, C.M. Kay, J. Am. Chem. Soc. 108 (1986) 2724-2727.

[16] F. Ascoli, R. Santucci, J. Inorg. Biochem. 68 (1996) 211-214.

[17] F. Sinibaldi, G. Mei, F. Polticelli, M.C. Piro, B.D. Howes, G. Smulevich, R. Santucci, F. Ascoli, L. Fiorucci, Protein Sci. 14 (2005) 1049-1058.

[18] A.A. Kapralov, I.V. Kumikov, I.I. Vlasova, N.A. Belikova, V.A. Tyurin, L.V. Basova, Q. Zhao, Y.Y. Tyurina, J. Jiang, H. Bayer, Y.A. Vladimirov, V.E. Kagan, Biochemistry 46 (2007) 14232-14244.

[19] T.L. Luntz, A. Schejter, E.A. Garber, E. Margoliash, Proc. Natl. Acad. Sci. USA 86 (1989) 3524-3528.

[20] Z.H. Wang, Y.W. Lin, F.I. Rosell, F.Y. Ni, H.J. Lu, P.Y. Yang, X.S. Tan, X.Y. Li, Z.X. Huang, A.G. Mauk, Chembiochem. 8 (2007) 607-609.

[21] T. Matsui, S. Ozaki, E. Liong, G.N. Phillips Jr., Y. Watanabe, J. Biol. Chem. 274 (1999) 2838-2844

[22] M. Garcia Fernandez, L. Troiano, L. Moretti, M. Nasi, M. Pinti, S. Salvioli, J. Dobrucki, A. Cossarizza, Cell Growth Differ. 13 (2002) 449-455.

[23] H.M. Gao, P.T. Kotzbauer, K. Uryu, S. Leight, J.Q. Trojanowski, V.M. Lee, J. Neurosci. 28 (2008) 7687-7698.

[24] V.E. Kagan, P. Wipf, D. Stoyanovsky, J.S. Greenberger, G. Borisenko, N.A. Belikova, N. Yanamala, A.K. Samhan Arias, M.A. Tungekar, J. Jiang, Y.Y. Tyurina, J. Ji, J. Klein-Seetharaman, B.R. Pitt, A.A. Shvedova, H. Bayir, Adv. Drug Deliv. Rev. 61 (2009) 1375-1385.

[25] H.S. Kim, Y.H. Suh, Behav. Brain Res. 196 (2009) 169-179.

[26] W. Noble, C.J. Garwood, D.P. Hanger, Prion 2 (2009) 78-83.

[27] V.W. Yong, J. Wells, F. Giuliani, S. Casha, C. Power, L.M. Metz, Lancet Neurol. 12 (2004) 744-751.

[28] E.K. Touminem, C.J. Wallace, P.K. Kinnunen, J. Biol. Chem. 277 (2002) 88228826.

[29] F. Sinibaldi, B.D. Howes, M.C. Piro, P. Caroppi, G. Mei, F. Ascoli, G. Smulevich, R. Santucci, J. Biol. Inorg. Chem. 11 (2006) 52-62.

[30] C. Purring-Koch, G. McLendon, Proc. Natl. Acad. Sci. USA 24 (2000) 1192811931.

[31] G.C. Brown, V. Borutaite, Biochim. Biophys. Acta 1777 (2008) 877-881. 